PROCEEDINGS OF THE

AMERICAN MATHEMATICAL SOCIETY

Volume 133, Number 9, Pages 2741-2750

S 0002-9939(05)07861-5

Article electronically published on March 29, 2005

\title{
CARDINAL RESTRICTIONS ON SOME HOMOGENEOUS COMPACTA
}

\author{
ISTVÁN JUHÁSZ, PETER NYIKOS, AND ZOLTÁN SZENTMIKLÓSSY
}

(Communicated by Alan Dow)

\begin{abstract}
We give restrictions on the cardinality of compact Hausdorff homogeneous spaces that do not use other cardinal invariants, but rather covering and separation properties. In particular, we show that it is consistent that every hereditarily normal homogeneous compactum is of cardinality $\mathfrak{c}$. We introduce property $\mathrm{wD}(\kappa)$, intermediate between the properties of being weakly $\kappa$-collectionwise Hausdorff and strongly $\kappa$-collectionwise Hausdorff, and show that if $X$ is a compact Hausdorff homogeneous space in which every subspace has property $\mathrm{wD}\left(\aleph_{1}\right)$, then $X$ is countably tight and hence of cardinality $\leq 2^{\mathfrak{c}}$. As a corollary, it is consistent that such a space $X$ is first countable and hence of cardinality $\mathfrak{c}$. A number of related results are shown and open problems presented.
\end{abstract}

\section{INTRODUCTION}

In this paper, "space" means "Hausdorff space" and "compactum" stands for "infinite compact (Hausdorff) space." It is well known that every compactum without isolated points is of cardinality $\geq \mathfrak{c}\left(=2^{\aleph_{0}}\right)$.

At the end of [vM], Jan van Mill posed the following problem:

1.1. Problem. Is every $T_{5}$ [that is, hereditarily normal] homogeneous compactum of cardinality $\mathfrak{c}$ ?

In a July 2003 seminar at the Alfred Rényi Institute in Budapest, he conjectured that the answer to his question was positive. In this paper, we show that it is consistent that the answer to van Mill's question is positive [Theorem 2.8]. We also show it is consistent [Theorem 3.2] that every homogeneous compactum that is hereditarily $\mathrm{wD}\left(\aleph_{1}\right)$ is first countable and hence, by a famous theorem of Arhangel'skiu, of cardinality $c$. This theorem says that $|X| \leq 2^{\chi(X)}$ for all compact Hausdorff $X$, where $\chi(X)$, as usual, stands for the character of $X$. When combined with the Cech-Pospišil theorem, this implies that every homogeneous compactum $X$ is exactly of cardinality $2^{\chi(X)}$.

Received by the editors January 1, 2004 and, in revised form, May 27, 2004.

2000 Mathematics Subject Classification. Primary 03E35, 54A25, 54D15, 54D30, 54F99; Secondary 03E50, 54D45.

Key words and phrases. Compactum, homogeneous, hereditarily, [weakly] [strongly] $\kappa$-cwH, property $\mathrm{wD}(\kappa), \mathrm{T}_{5}$, tightness, $\pi$-character, $\pi$-base, first countable, $G_{\delta}$.

Research of the first and third authors partially supported by OTKA grant no. 37758.

Research of the second author partially supported by a grant from the Erdös Center of the János Bolyai Mathematical Society.

(C)2005 American Mathematical Society Reverts to public domain 28 years from publication 
1.2. Definition. Let $\kappa$ be a cardinal number. A space $X$ is weakly $\kappa$-collectionwise Hausdorff [resp. satisfies property $w D(\kappa)$ ] if every closed discrete subspace $D$ of cardinality $\kappa$ has a subset $D_{0}$ of cardinality $\kappa$ which can be expanded to a disjoint [resp. discrete] collection of open sets $U_{d}$ such that $U_{d} \cap D_{0}=\{d\}$ for all $d \in D_{0}$.

We use " $\kappa$-cwH" as an abbreviation for " $\kappa$-collectionwise Hausdorff": without the modifier "weakly" this means that $D_{0}$ can be taken to be $D$ in the above definition.

1.3. Definition. A space $X$ is [strongly] $\kappa$-cwH if every closed discrete subspace $D$ of cardinality $\leq \kappa$ can be expanded to a disjoint [resp. discrete] collection of open sets as in Definition 1.2.

In Cohen's original model for the negation of the Continuum Hypothesis $(\mathrm{CH})$, and indeed in any model obtained by adding $\aleph_{2}$ Cohen reals to a model of ZFC, every locally compact normal space is $\aleph_{1}$-cwH. This well-known fact can be shown by applying the proof technique of [W] Theorem 3] to [DTW, Theorem 1.4].

It is also well known and easy to prove that every normal $\kappa$-cwH space is strongly $\kappa$-cwH; similarly, every normal, weakly $\kappa$-cwH space has property $\mathrm{wD}(\kappa)$. Therefore, every $\mathrm{T}_{5}$, locally compact space is hereditarily strongly $\omega_{1}$-cwH and hence hereditarily $\mathrm{wD}\left(\aleph_{1}\right)$ in any generic extension obtained by adding $\aleph_{2}$ Cohen reals to an arbitrary ground model. This fact figures prominently in the proof of Theorem 2.8 that yields a consistent Yes answer to Problem 1.1.

With one exception that we know of, the theorems in this paper represent the first restrictions on the cardinality of homogeneous compacta that do not assume restrictions on other well-known cardinal functions (character, cellularity, tightness, etc.). The exception is that every monotonically normal homogeneous compactum is first countable (and therefore of cardinality $\mathfrak{c}$ ). This has been part of the folklore for some time, but we give a quick proof below (Theorem 3.6). Monotone normality is a very strong property, and Problem 1.1 asks whether at least the weaker part of this result can be extended to the much wider class of $\mathrm{T}_{5}$ homogeneous compacta. Perhaps (see Problems 3.4 and 3.5 below) the stronger part can be extended also.

\section{A Dichotomy And A COnsistency Result for Problem 1.1}

We begin with a lemma which we hope to be of independent interest. Recall that a (local) $\pi$-base for a point $x$ of a space $X$ is a family $\mathcal{U}$ of nonempty open sets such that every neighbourhood of $x$ contains a member of $\mathcal{U}$. The least cardinality of a $\pi$-base for $x$ is called the $\pi$-character of $x$ in $X$ and is denoted by $\pi \chi(x, X)$.

2.1. Lemma. Let $Y$ be a locally compact space. The set of points y which fail to satisfy at least one of the following two properties is dense in $Y$.

(a) $\pi \chi(y, Y)=\omega$.

(b) Every $G_{\delta}$ containing y has nonempty interior.

In particular, if $Y$ has both points satisfying (a) and points satisfying (b), then $Y$ cannot be homogeneous.

Proof. If $Y$ has a dense set of isolated points, then $(a)$ fails for these and we are done. [Some papers use the convention that $\pi \chi(y, Y)=\omega$ even if $y$ is isolated, but not this paper.] Otherwise, let $A$ be the closure of the set of isolated points. It is enough to show that the (locally compact) subspace $Y \backslash A$ has a dense set of points where at least one of $(a)$ or $(b)$ fails. In other words, it only remains to prove our 
lemma for the case where $Y$ has no isolated points, and we assume this from now on.

Let $U_{0}$ be any nonempty open subset of $Y$ with compact closure. We define a descending well-ordered family of such sets by induction. If $\alpha$ is an ordinal and $U_{\alpha}$ has been defined, let $U_{\alpha+1}$ be any nonempty open set whose closure is a proper subset of $U_{\alpha}$.

If $\alpha$ is a limit ordinal and $U_{\xi}$ has been defined for all $\xi<\alpha$, then $C=\bigcap\left\{U_{\xi}\right.$ : $\xi<\alpha\}$ is nonempty because it is the intersection of the chain of compact sets $\left\{\overline{U_{\xi}}: \xi<\alpha\right\}$. If $\alpha$ is of countable cofinality and $y$ is a point of $C$ satisfying $(b)$, then $C$, being a $G_{\delta}$, has nonempty interior and we may let $U_{\alpha}$ be the interior of $C$. If $\alpha$ is of uncountable cofinality and $y$ is a point of $C$ satisfying $(a)$, then let $\left\{V_{n}: n \in \omega\right\}$ be a countable $\pi$-base for $y$. Since $\alpha$ is of uncountable cofinality, there must exist $n$ such that $V_{n} \subset U_{\xi}$ for cofinally many $\xi \in \alpha$, but then $V_{n} \subset U_{\xi}$ for all $\xi \in \alpha$ because the $U_{\xi}$ 's are nested, hence $V_{n} \subset C$. So now we can let $U_{\alpha}=V_{n}$ and continue the induction.

Eventually the chain stops at some limit ordinal $\alpha$, i.e. the intersection $C$ has empty interior. But then all the points of $C$ fail to satisfy $(b)$ if $\alpha$ is of countable cofinality, and fail to satisfy $(a)$ if $\alpha$ is of uncountable cofinality. Since $U_{0}$ was arbitrary, this completes the proof.

2.2. Corollary. If $Y$ is a locally compact space in which every point is nonisolated and of countable $\pi$-character, then the union of the closed nowhere dense $G_{\delta}$-subsets of $X$ is dense in $X$.

By a theorem of Shapirovskil, every compact $T_{5}$ space has a point of countable $\pi$-character, so that (a) in Lemma 2.1 is automatically satisfied by some points of any $\mathrm{T}_{5}$ compactum. This theorem of Shapirovskil is a corollary of his remarkable theorem that a compactum cannot be mapped continuously onto $[0,1]^{\aleph_{1}}$ iff every subcompactum has a point of countable relative $\pi$-character. This gives us many classes of compacta with points of countable $\pi$-character, including the class of countably tight compacta. Two other classes are given by the following two theorems, which seem to be new.

2.3. Theorem. If $X$ is a hereditarily weakly $\omega_{1}$-cwH compact space, then $X$ cannot be mapped continuously onto $[0,1]^{\aleph_{1}}$.

Proof. Suppose $f: X \rightarrow[0,1]^{\aleph_{1}}$ is a continuous onto function. Let $F$ be a closed subset of $X$ such that the restriction of $f$ to $F$ is irreducible; that is, no proper closed subset of $F$ maps onto $[0,1]^{\aleph_{1}}$. Let $Q$ be a countable dense subset of $[0,1]^{\aleph_{1}}$. By irreducibility, a subset of $F$ which meets each point-inverse $f^{-1}(q)(q \in Q)$ is dense in $F$; hence $F$ is separable. By the hereditarily weakly $\omega_{1}$-cwH property, $F$ cannot have an uncountable discrete subspace; but $[0,1]^{\aleph_{1}}$ does have one, and this contradicts the surjectivity of $f \uparrow F$.

An alternative proof of Theorem 2.3 uses the fact that $[0,1]^{\aleph_{1}}$ satisfies the countable chain condition, and that a closed irreducible map has the property that a pair of disjoint nonempty open sets in the domain has images with nonempty disjoint interiors.

2.4. Theorem. If $X$ is a compact space satisfying $w D\left(\aleph_{0}\right)$ hereditarily, then $X$ cannot be mapped continuously onto $[0,1]^{\aleph_{1}}$. 
Proof. Let $Y$ be the Tychonoff plank, $\left(\omega_{1} \times \omega+1\right) \cup\left(\omega_{1}+1 \times \omega\right)$, let $S$ be a copy of $[0,1]^{\aleph_{1}}$ that contains $Y$, and let $f: X \rightarrow S$ be a continuous onto function. Let $Z=f^{-1} Y$ and for each point $\langle\alpha, n\rangle$ in $\omega_{1} \times \omega$, let $x(\alpha, n) \in f^{-1}\{\langle\alpha, n\rangle\}$. Since the preimage of $\left(\omega_{1}+1 \times\{n\}\right)$ is compact, there is a condensation point $z_{n}$ of $\left\{x(\alpha, n): \alpha \in \omega_{1}\right\}$, and $z_{n} \in f^{-1}\left\{\left\langle\omega_{1}, n\right\rangle\right\}$. Clearly $\left\{z_{n}: n \in \omega\right\}$ is closed and discrete in $Z$.

Suppose $A$ is an infinite subset of $\omega$ and $\left\{U_{n}: n \in A\right\}$ is a family of open sets satisfying $z_{n} \in U_{n}$ for all $n \in A$. For every $n \in A$, the closure of $U_{n}$ contains the preimage of $C_{n} \times\{n\}$ for some club subset $C_{n}$ of $\omega_{1}$. Fix $\alpha \in \bigcap_{n \in A} C_{n}$ and let $x_{n} \in f^{-1}\{\langle\alpha, n\rangle\} \cap \overline{U_{n}}$ for all $n \in A$. Since the preimage of $\{\alpha\} \times \omega+1$ is a compact subset of $Z$, there is an accumulation point of $\left\{x_{n}: n \in A\right\}$, which shows that $\left\{U_{n}: n \in A\right\}$ is not a discrete collection.

2.5. Corollary. If $X$ is a compact space that is either hereditarily weakly $\omega_{1}$ $c w H$ or hereditarily $w D\left(\aleph_{0}\right)$, then $X$ has a dense set of points of countable $\pi$ character.

The condition on discrete subsets that appears in our next lemma is clearly satisfied by any Lindelöf space. Further information on it will be given by Theorem 3.13 .

2.6. Lemma. Let $X$ be a hereditarily $w D\left(\aleph_{1}\right)$ space, such that every discrete subset of $X$ of cardinality $\aleph_{1}$ has a complete accumulation point. Let $D$ be a discrete subset of $X$, of cardinality $\aleph_{1}$, and let $H$ be a closed $G_{\delta}$-subset of $X$ containing all the complete accumulation points of $D$. Then $H$ has nonempty interior.

Proof. Obviously, $D \backslash H$ is countable. Let $Y=X \backslash(D \cap H)^{\prime}$, where $(D \cap H)^{\prime}$ stands for the derived set of all limit points of $D \cap H$. Since $D$ is discrete, $D \cap H$ is a closed discrete subset of $Y$ of cardinality $\aleph_{1}$. Let $\mathcal{U}$ be a discrete (in $Y$ ) family of $\aleph_{1}$ open subsets of $Y$ expanding $\aleph_{1}$ points of $D \cap H$. Since $H$ is closed, $(D \cap H)^{\prime} \subset H$ and so $Y \backslash H=X \backslash H$ is a countable union of closed subsets $F_{n}(n \in \omega)$ of $X$. So all but countably many members of $\mathcal{U}$ are subsets of $H$ : otherwise, we could pick points of uncountably many of them in some fixed set $F_{n}$ and thus have an uncountable closed discrete subset of $F_{n}$ and hence of $X$.

2.7. Theorem. If $X$ is a homogeneous compact space satisfying $w D\left(\aleph_{1}\right)$ hereditarily, then $X$ is countably tight and hence of cardinality $\leq 2^{\mathfrak{c}}$.

Proof. If $X$ is of uncountable tightness, then by the main theorem of [JSz, $X$ has a free $\omega_{1}$-sequence converging to some point $y$. A free $\omega_{1}$-sequence in a space $X$ is a family $\left\{x_{\alpha}: \alpha<\omega_{1}\right\}$ of points such that, for each $\alpha<\omega_{1}$, the closure of $\left\{x_{\xi}: \xi<\alpha\right\}$ does not meet the closure of $\left\{x_{\eta}: \eta \geq \alpha\right\}$. It is easily seen that every free sequence is a discrete subspace. To say that it converges to $y$ is to say that every neighbourhood of $y$ contains all but countably many of its points. Equivalently, $y$ is the only complete accumulation point of $\left\{x_{\alpha}: \alpha<\omega_{1}\right\}$. Now, every $G_{\delta}$ containing $y$ shrinks to a closed $G_{\delta}$ that also contains $y$ by regularity of $X$, and so, by Lemma 2.6, has nonempty interior. Consequently, $y$ satisfies condition (b) in Lemma 2.1. However, this contradicts the homogeneity of $X$ because of Corollary 2.5 and Lemma 2.1.

For the second conclusion, we use the theorem in [J2] that every compact space of countable tightness has a point of character $\leq \mathfrak{c}$. By homogeneity, this implies every point is of character $\leq \mathfrak{c}$, and so $|X| \leq 2^{\mathfrak{c}}$ by the Arhangel'skiı̌ Theorem. 
We are now ready to give the consistent affirmative answer to Problem 1.1 promised in the introduction.

2.8. Theorem. If (at least) $\aleph_{2}$ Cohen reals are added to any model $V$ of $Z F C$, the resulting extension $W$ has the property that every homogeneous $T_{5}$ compactum is countably tight and of character $\leq \aleph_{1}$, hence of cardinality $\leq 2^{\aleph_{1}}$. In particular, if $\left(2^{\aleph_{1}}\right)^{V}$ Cohen reals are added, then in the resulting extension every homogeneous, countably tight compactum, hence every homogeneous $T_{5}$ compactum, is of cardinality $\mathfrak{c}$.

Proof. By the remarks following Definition 1.3, every homogeneous compact $\mathrm{T}_{5}$ space in $W$ satisfies property $\mathrm{wD}\left(\aleph_{1}\right)$ hereditarily, and hence is countably tight by Theorem 2.7. Moreover, in JJ2 it is shown that every compact space of countable tightness has a point of character $\leq \aleph_{1}$ in an extension obtained by adding just $\aleph_{1}$ Cohen reals to an arbitrary model of ZFC. [It is also conjectured there that this may actually be true in ZFC.] But first adding $\aleph_{2}$ Cohen reals and then adding $\aleph_{1}$ is equivalent to adding $\aleph_{2}$ Cohen reals all at once. Thus if one adds (at least) $\aleph_{2}$ Cohen reals, then in the resulting extension every homogeneous compact space of countable tightness is of character $\leq \aleph_{1}$, hence of cardinality $\leq 2^{\aleph_{1}}$ by the Arhangel'skiu Theorem. Finally, if $\left(2^{\aleph_{1}}\right)^{V}$ Cohen reals are added, then the resulting model of ZFC also satisfies $\mathfrak{c}=2^{\aleph_{1}}$.

\section{Obtaining First COUntability in SOME homogeneous COMPACTA}

Arhangel'skiǔ has conjectured [ $\underline{\mathrm{A}}$ that every homogeneous, countably tight compactum is of cardinality $\mathfrak{c}$. Theorem 2.8 proves the consistency of this, but the conjecture is also a consequence of the Proper Forcing Axiom (PFA), which implies that every countably tight compactum has a point of first countability [D1, J3. Theorem 3.3].

Our next theorem uses a different, much weaker axiom to get first countability of homogeneous, hereditarily $\mathrm{wD}\left(\aleph_{1}\right)$ compacta. For convenience, we adopt the following terminology.

Axiom I. Every locally compact space of countable spread has a point of first countability.

Axiom L. Every locally compact space of countable spread is hereditarily Lindelöf.

It makes no difference whether we leave out "locally" in either axiom, since countable spread (viz., the property that every discrete subspace is countable), the hereditary Lindelöf property, and the existence of a point of first countability are obviously preserved on taking the one-point compactification. Going in the opposite direction, one observes that in a locally compact space, a point has a countable local base iff it is a $G_{\delta}$, and every point of a locally compact space is contained in a compact $G_{\delta}$. Also, since every point is a $G_{\delta}$ in a regular hereditarily Lindelöf space, Axiom L implies Axiom I.

A well-known old result of the third author is that Axiom L is true under MA $\left(\omega_{1}\right)$ $[\mathrm{Sz},[\mathrm{R}, 6.4]$. It is also true in various models obtained from a countable support iteration of forcing with all ccc [or all proper] posets that do not destroy a certain coherent Souslin tree and then forcing with the tree. However, we do not know if Axiom I is strictly weaker than Axiom L. 
Before giving the theorem using Axiom I, however, we need to prove the following ZFC result.

3.1. Lemma. In a locally compact, hereditarily $w D\left(\aleph_{1}\right)$ space, the boundary of any open Lindelöf subset has countable spread.

Proof. Let $H$ be an open Lindelöf subset of a space $X$ as described. Let $D$ be a discrete subspace of the boundary of $H$; since $H$ is open, its boundary is $\bar{H} \backslash H$. Let $D^{\prime}$ be the derived set of $D$; since $D$ is discrete, $D^{\prime}=\bar{D} \backslash D$. Also, $D^{\prime}$ is disjoint from $H$ because $\bar{H} \backslash H$ is closed. Hence $H$ is a subset of $W=\bar{H} \backslash D^{\prime}$, and $D$ is closed in the relative topology of $W$.

If $D$ were uncountable, there would be an uncountable subset $D_{0}$ of $D$ with a discrete-in- $W$ open expansion $\left\{U_{d}: d \in D_{0}\right\}$. But then $\left\{U_{d} \cap H: d \in D_{0}\right\}$ would be an uncountable discrete-in- $H$ collection of subsets of $H$, and this is a contradiction since $H$ is Lindelöf. Hence $D$ is countable.

3.2. Theorem. Assume Axiom I. Then every homogeneous, hereditarily $w D\left(\aleph_{1}\right)$ compactum is first countable and hence of cardinality $\mathfrak{c}$.

Proof. Let $X$ be a compactum as above. By homogeneity and Corollaries 2.2 and 2.5, every point in $X$ is contained in a nowhere dense closed $G_{\delta}$ set $B$. The complement of $B$ is an open Lindelöf subspace whose boundary is $B$, and so $B$ is of countable spread by Lemma 2.6. Now we use Axiom I to conclude that $B$ has a point $x$ which is a relative $G_{\delta}$-subset of $B$. But since $B$ is itself a $G_{\delta}$-subset of $X$, it follows that $x$ is a $G_{\delta}$-point of $X$ and hence a point of first countability. Another application of homogeneity gives us first countability of $X$.

Although Axiom I does not hold in the models obtained by adding Cohen reals, the following conjecture seems reasonable in light of Theorems 2.8 and 3.2.

3.3. Conjecture. It is consistent that every homogeneous $T_{5}$ compactum is first countable.

Jan van Mill has observed that in any model of $2^{\aleph_{0}}<2^{\aleph_{1}}$, a Yes answer to Problem 1.1 would imply that every $\mathrm{T}_{5}$ homogeneous compactum is first countable, since every compact space of cardinality $<2^{\aleph_{1}}$ has a point of first countability by the Cech-Pospišil Theorem. Of course, the models of Theorem 2.8 that do give a Yes answer to Problem 1.1 satisfy $2^{\aleph_{0}}=2^{\aleph_{1}}$. At the moment, the best candidate for confirmation of our conjecture is one of the models whose construction involves a coherent Souslin tree. Positive answers to both of the following questions in the same model of set theory would also confirm the conjecture:

3.4. Problem. Is every homogeneous $T_{5}$ compactum countably tight?

3.5. Problem. Is every homogeneous countably tight compactum first countable?

As we have seen, the PFA implies a positive answer to Problem 3.5, and the answer to Problem 3.4 is positive in models from Theorem 2.8, but we would also like to have it the other way around!

As indicated in the introduction, Conjecture 3.3 is provable in ZFC for monotonically normal compacta.

3.6. Theorem. Every monotonically normal homogeneous compactum is first countable and hence of cardinality $\mathfrak{c}$. Moreover, it is hereditarily paracompact. 
Proof. This is a quick corollary of the following theorem of Williams and Zhou [WZ], [J3, 3.12]:

Let $P(p, X)$ denote $\min \left\{\kappa: p\right.$ is not a $\mathrm{P}_{\kappa^{+}}$-point of $\left.X\right\}$ (i.e., $P(p, X)$ is the smallest size of a family of neighbourhoods of $p$ in $X$ whose intersection is not a neighbourhood of $p$ ). Then in a monotonically normal compact space $X$, the set of all points $p$ such that $\chi(p, X)=P(p, X)$ is dense in $X$.

Since a compact $\mathrm{P}$-space is finite, $P(p, X)$ is countable for all points of a compact homogeneous monotonically normal space, and so the space is first countable.

For the hereditary paracompactness, just use the Balogh-Rudin theorem that a monotonically normal space is hereditarily paracompact iff it contains no homeomorphic copy of a stationary subset of $\omega_{1}[\mathrm{BR}$, and the elementary fact that no first countable compact space can contain such a copy.

If we assume $\operatorname{MA}\left(\omega_{1}\right)+$ Axiom $\mathrm{R}$, we can relax "monotonically normal" in Theorem 3.6 to "hereditarily strongly $\omega_{1}$-cwH." [Recall that every monotonically normal space is hereditarily collectionwise normal.] Theorem 3.2 gives us first countability since $\mathrm{MA}\left(\omega_{1}\right)$ implies Axiom I, and hereditary paracompactness follows from Balogh's theorem $\left[\mathrm{B}\right.$ that MA $\left(\omega_{1}\right)+$ Axiom R implies every hereditarily strongly $\omega_{1}-\mathrm{cwH}$ space is either hereditarily paracompact or contains a perfect preimage of $\omega_{1}$.

The following question lies at the opposite extreme of generality. It is completely open - we have no consistency results in either direction.

3.7. Problem. Is every homogeneous compactum either first countable or continuously mappable onto $[0,1]^{\aleph_{1}}$ ?

For homogeneous ccc (that is, countable cellularity) compacta the answer is Yes if one assumes $2^{\aleph_{0}}<2^{\aleph_{1}}$. This is because $|X| \leq 2^{c(X) \pi \chi(X)}$ for homogeneous spaces A], and so any homogeneous ccc compactum of cardinality $>\mathfrak{c}$ is also of uncountable $\pi$-character and hence continuously mappable onto $[0,1]^{\aleph_{1}}$ by Shapirovskilı's theorem. On the other hand, since $|X|=2^{\chi(X)}$ for every homogeneous compactum, $2^{\aleph_{0}}<2^{\aleph_{1}}$ implies that every homogeneous compactum of cardinality $\mathfrak{c}$ is first countable. This reasoning extends to higher cardinals, and results in such considerations as the following: if, as some suspect, every homogeneous compactum is of cellularity $\leq \mathfrak{c}$, then $2^{\mathfrak{c}}<2^{\mathfrak{c}^{+}}$implies that every homogeneous compactum is either of character $\leq \mathfrak{c}$ or admits a continous map onto $[0,1]^{\mathfrak{c}^{+}}$.

Problem 3.7 is closely related to the following general problem about compacta.

3.7'. Problem. Is it consistent that every compactum either has a point of first countability or admits a continuous map onto $[0,1]^{\aleph_{1}}$ ?

There are compacta satisfying neither condition in various models of set theory, including those satisfying $\mathrm{CH}[\mathrm{F}]$ and those satisfying $\boldsymbol{p}$ or its weakening $(t)$, which holds whenever a single Cohen real is added [J1].

Our next theorem features a use of the property that every open Lindelöf subspace has hereditarily Lindelöf boundary. This property is satisfied by every locally compact space satisfying $\mathrm{wD}\left(\aleph_{1}\right)$ hereditarily in any model of ZFC where Axiom $\mathrm{L}$ holds, by Lemma 3.1. It is already strong enough to imply first countability in any homogeneous compactum [Theorem 3.11]. We will make use of two lemmas, the first of which is very easy (cf. $\mathrm{Ny}]$ or $\mathrm{B}]$ ). 
3.8. Lemma. In a locally compact space, every point has an open Lindelöf (equivalently: $\sigma$-compact) neighbourhood.

3.9. Lemma. Let $X$ be a locally compact space such that every Lindelöf subset has Lindelöf closure. Then any two disjoint closed subsets of $X$, one of which is Lindelöf, can be put into disjoint open sets. Hence $X$ has Property $w D\left(\aleph_{0}\right)$.

Proof. Let $A$ and $B$ be disjoint closed subsets of $X$. If $A$ is Lindelöf, then Lemma 3.8 gives a countable cover $\mathcal{U}$ of $A$ by open Lindelöf sets, none of which meets $B$. Then $U=\bigcup \mathcal{U}$ is Lindelöf, and so $A$ and $B \cap \bar{U}$ are disjoint closed Lindelöf subsets of $X$ and hence can be put into disjoint open subsets $V$ and $W$ of $X$. Then $V$ and $W \cup(X \backslash \bar{U})$ are disjoint open subsets of $X$ containing $A$ and $B$, respectively.

For the final conclusion, note that $X$ is pseudonormal (that is, every pair of disjoint closed subsets, one of which is countable, can be put into disjoint open sets) and use the easy fact $\mathrm{vD}, 12.1]$ that every pseudonormal space satisfies $\mathrm{wD}\left(\aleph_{0}\right)$.

Before going on to Theorem 3.11, we note an interesting "stepping-down" consequence of Lemmas 3.1 and 3.9 in the presence of Axiom L.

3.10. Corollary (Axiom L). Let $X$ be a locally compact space. If $X$ is hereditarily $w D\left(\aleph_{1}\right)$, then $X$ is hereditarily $w D\left(\aleph_{0}\right)$.

Proof. First, note that the property of being $\mathrm{wD}(\kappa)$ is inherited by all subspaces if it is inherited by all open subspaces. This is because, if $Y$ is a subspace of $X$ and $D$ is a closed discrete subspace of $Y$, then $D$ is also closed and discrete in the (open, in $X$ ) subspace $X \backslash D^{\prime}$, where $D^{\prime}$ again stands for the derived set of $D$ in $X$. So if we define $D_{0}$ and the sets $U_{d}$ as in Definition 1.2, with $X \backslash D^{\prime}$ playing the role of $X$, then the traces $U_{d} \cap Y$ will be a discrete collection of open subsets of $Y$ expanding $D_{0}$ in $Y$, as desired.

Next, let $Y$ be an open subspace of $X$. Since $Y$ is locally compact, it follows from Lemma 3.1 and Axiom L that every open subspace of $Y$ has hereditarily Lindelöf boundary, and hence that every Lindelöf subspace of $Y$ has Lindelöf closure. Now use Lemma 3.9 to conclude that $Y$ has Property $\mathrm{wD}\left(\aleph_{0}\right)$.

Corollary 3.10 requires more than just ZFC. It is easy to modify Ostaszewski's space using $\diamond$ to get a hereditarily separable locally compact locally countable space which is not $\mathrm{wD}\left(\aleph_{0}\right)$; yet it is vacuously (hereditarily) $\mathrm{wD}\left(\aleph_{1}\right)$. Details will be given in $\mathrm{NyP}$ along with a simple ZFC example to show that local compactness cannot be dropped from Corollary 3.10.

3.11. Theorem. Let $K$ be a homogeneous compactum in which every open Lindelöf subspace has hereditarily Lindelöf boundary. Then $K$ is first countable.

Proof. Let $p \in K$ and let $X=K \backslash\{p\}$. If $X$ is Lindelöf, then $p$ is a $G_{\delta}$-point in $K$, hence a first countability point, and we are done. So we may assume that $X$ is not Lindelöf.

Next we show $K$ satisfies $\mathrm{wD}\left(\aleph_{0}\right)$ hereditarily. Indeed, the property that every open Lindelöf subspace of $K$ has hereditarily Lindelöf boundary is inherited by open subspaces of $K$. It follows that if $Y$ is an open subspace of $K$, then every Lindelöf subspace of $Y$ has Lindelöf closure; hence $Y$ satisfies $\mathrm{wD}\left(\aleph_{0}\right)$, and so every subspace of $K$ satisfies it (see the first paragraph in the proof of Corollary 3.10). 
From Corollary 2.5 we get that $K$ has a dense set of points of countable $\pi$ character. Since $p$ is nonisolated, $X$ is noncompact and so contains a noncompact, Lindelöf open subset $X_{0}$, as can be easily shown by repeated applications of Lemma 3.8. Continue inductively through the countable ordinals, defining a strictly increasing chain of Lindelöf open subsets of $X$ as follows. We get $X_{\xi+1}$ once $X_{\xi}$ is defined by covering the boundary of $X_{\xi}$ with countably many open Lindelöf subspaces of $X$. Note that $X_{\xi+1}$ can be chosen to be strictly larger than $c \ell_{X}\left(X_{\xi}\right)$; this is where we use the assumption that $X$ is not Lindelöf. At limit ordinals $\alpha$ let $X_{\alpha}=\bigcup\left\{X_{\xi}: \xi<\alpha\right\}$. By induction, $X_{\xi}$ is Lindelöf and has hereditarily Lindelöf boundary for all countable $\xi$.

Having finished the induction, $X_{\omega_{1}}=\bigcup\left\{X_{\xi}: \xi<\omega_{1}\right\}$ is a non-Lindelöf open subset of $X$. If $X_{\omega_{1}}$ is clopen in $X$, then for every open neighbourhood $U$ of $p$ in $K$ the compact set $X_{\omega_{1}} \backslash U$ is contained in $X_{\alpha}$ for some $\alpha<\omega_{1}$. Consequently, every $G_{\delta}$-set containing $p$ contains $X_{\omega_{1}} \backslash X_{\alpha}$ for some $\alpha<\omega_{1}$ and so has nonempty interior. This means that $p$ can play the role of $y$ in Lemma $2.1(b)$. Otherwise, since $X$ satisfies $\mathrm{wD}\left(\aleph_{0}\right)$ hereditarily, any point on the boundary of $X_{\omega_{1}}$ in $X$ is isolated in $B d_{X}\left(X_{\omega_{1}}\right)[\mathrm{Ny}$, Lemma 1.6], and so can play the role of $y$ in (b) similarly to $p$ in the case where $X_{\omega_{1}}$ is clopen in $X$. This, by Lemma 2.1, contradicts the homogeneity of $K$ and shows that $X$ is indeed Lindelöf.

Our next problem involves condition (b) in Lemma 2.1, which in a homogeneous space is equivalent to every nonempty $G_{\delta}$ having nonempty interior. Spaces with this latter property are often called pseudo-P spaces.

\subsection{Problem. Is there a homogeneous pseudo-P compactum?}

The Stone-Čech remainder of $\omega$ is a pseudo-P compactum, but is well known not to be homogeneous. Jan van Mill has observed that any pseudo-P compactum of weight $\leq \omega_{1}$ has a dense set of P-points (just use W. Rudin's argument that $\mathrm{CH}$ implies $\beta \omega-\omega$ has P-points) and so an example for Problem 3.12 would have to have weight $>\omega_{1}$.

Finally, here is an independent result about the hypothesis used in Lemma 2.6. Recall that an $S$-space is a hereditarily separable regular space that is not hereditarily Lindelöf. The existence of an S-space is ZFC-independent $[\mathbb{R}]$.

3.13. Theorem. The following axioms are equivalent.

(1) There is an S-space.

(2) There is a regular locally countable uncountable space of countable spread.

(3) There is a regular space $X$ in which every discrete subspace of cardinality $\aleph_{1}$ has a complete accumulation point, yet there is a subspace of cardinality $\aleph_{1}$ without a complete accumulation point.

Proof. (1) $\Longrightarrow(2)$ is well known [cf. the proof of Theorem 3.1 in $[\mathbf{R}]$, and $(2) \Longrightarrow(3)$ is obvious. To see (3) $\Longrightarrow(1)$, take $Y \subset X$ of size $\aleph_{1}$ with no complete accumulation point. Then $Y$ must have countable spread and is not Lindelöf, hence it contains an uncountable right separated subspace $Z$. But then $Z$ is an S-space [R, proof of 3.3].

\section{ADDED IN PROOF}

Ramiro de la Vega has shown (unpublished preprint) that Conjecture 3.3 is correct by showing in ZFC that every homogeneous countably tight compactum 
is of cardinality $\mathfrak{c}$. In particular, the conjecture holds if at least $\aleph_{2}$ Cohen reals are added to a model of ZFC, and the forcing extension satisfies $2^{\aleph_{0}}<2^{\aleph_{1}}$. (See Theorem 2.8 and the discussion of Problem 3.7.)

\section{REFERENCES}

[A] A. V. Arhangel'skiı̌, "Topological homogeneity. Topological groups and their continuous maps," Uspekhi Mat. Nauk 42 (2)(254) (1987) 69-105, 287. MR0898622 (89b:54004)

[B] Z. Balogh, "Locally nice spaces and axiom R," Topology Appl. 125 (2) (2002) 335-341. MR.1933581 (2003i:54029)

[BR] Z. Balogh and M.E. Rudin, "Monotone normality," Top. Appl. 47 (2) (1992), 115-127. MR.1193194 (94b:54065)

[vD] E.K. van Douwen, "The integers and topology," in: Handbook of Set-Theoretic Topology, K. Kunen and J. Vaughan, eds., North-Holland (1984) 111-167. MR.0776622 (87f:54008)

[D1] A. Dow, "An introduction to applications of elementary submodels to topology," Topology Proceedings 13 (1) (1988), 17-72. MR1031969 (91a:54003)

[D2] A. Dow, "Compact spaces of countable tightness in the Cohen model," in: Set theory and its applications (Toronto, ON, 1987), J. Steprans and S. Watson, eds., Lecture Notes in Mathematics \#1401, Springer-Verlag, (1989) 55-67. MR1031765|(91a:54004)

[DTW] A. Dow, F.D.Tall, and W.A.R. Weiss, "New proofs of the consistency of the normal Moore space conjecture I," Topology Appl. 37 (1) (1990), 33-51. MR1075372 (92b:54008a)

[F] V. V. Fedorchuk, "Bicompacta in which each infinite closed subset is n-dimensional," Math. USSR Sbornik 25 (1975) 37-57.

[J1] I. Juhász, "A weakening of $\mathbf{p}$, with applications to topology" Comment. Math. Univ. Carolinae 29 (1988), no. 4, 767-773. MR0982796 (90d:54005)

[J2] I. Juhász, "On the minimum character of points in compact spaces," in: Topology. Theory and applications, II (Pécs, 1989), Colloq. Math. Soc. János Bolyai, 55, North-Holland, Amsterdam, (1993) 365-371. MR1244377 (94k:54004)

[J3] I. Juhász, "Cardinal functions," in: Recent Progress in General Topology, M. Hušek and J. van Mill, eds., Elsevier, (1992), 417-441. MR.1229134

[JSz] I. Juhász and Z. Szentmiklóssy, "Convergent free sequences in compact spaces, Proc. Amer. Math. Soc. 116 (4) (1992), 1153-1160. MF.1137223 (93b:54024)

[vM] J. van Mill, "On the cardinality of power homogeneous compacta," preprint.

[Ny] P. Nyikos, "Applications of some strong set-theoretic axioms to locally compact $T_{5}$ and hereditarily scwH spaces," Fund. Math. 176 (1) (2003) 25-45. MR 1971471(2004k:54008)

[NyP] P. Nyikos and J.E. Porter, "Hereditarily strongly cwH and separation axioms," in preparation. Preliminary draft: www.math.sc.edu/ nyikos/preprints.html

[R] J. Roitman, "Basic S and L," in: Handbook of Set-Theoretic Topology, K. Kunen and J. Vaughan, eds., North-Holland (1984) 295-326. MF0776626 (87a:54043)

[Sz] Z. Szentmiklóssy, "S spaces and L spaces under Martin's axiom," Coll. Math. Soc. J. Bolyai, 23 (1978), II, 1139-1145. MR0588860 (81k:54032)

[W] W.S. Watson, "Locally compact normal spaces in the constructible universe," Canad. J. Math. 34 (5) (1982), 1091-1096. MR0675681 (83k:54021)

[WZ] S.W. Williams and H. Zhou, "Order-like structure of monotonically normal spaces," Comment. Math. Univ. Carolinae 39 (1) (1998), 207-217. MR:1623026|(99c:54029)

Alfred Rényi Institute, P.O. Box 127, 1364 Budapest, Hungary

Department of Mathematics, University of South Carolina, Columbia, South CarOLINA 29208

Department of Mathematics, Eötvös Loránd University, Pázmány sétány 1/C, BuDAPEST, H-1117 HuNGARY 